\title{
Sequential evaluation of CALR mutant burden in patients with myeloproliferative neoplasms
}

\author{
Chiara Cavalloni ${ }^{1, *}$, Elisa Rumi ${ }^{1,2, *}$, Virginia V. Ferretti ${ }^{2}$, Daniela Pietra ${ }^{2}$, Elisa \\ Roncoroni ${ }^{2}$, Marta Bellini ${ }^{1}$, Michele Ciboddo ${ }^{1}$, Ilaria C. Casetti ${ }^{1}$, Benedetta Landini ${ }^{2}$, \\ Elena Fugazza², Daniela Troletti ${ }^{2}$, Cesare Astori' ${ }^{2}$, Mario Cazzola ${ }^{1,2}$ \\ ${ }^{1}$ Department of Molecular Medicine, University of Pavia, Pavia, Italy \\ ${ }^{2}$ Department of Hematology Oncology, Fondazione Istituto di Ricovero e Cura a Carattere Scientifico (IRCCS) Policlinico San \\ Matteo, Pavia, Italy \\ *These authors contributed equally to this work
}

Correspondence to: Elisa Rumi, email: elisarumi@hotmail.com, elisa.rumi@unipv.it

Keywords: myeloproliferative, burden, CALR, JAK2, sequential

Received: January 21, 2017 Accepted: March 24, $2017 \quad$ Published: April 03, 2017

Copyright: Cavalloni et al. This is an open-access article distributed under the terms of the Creative Commons Attribution License (CC-BY), which permits unrestricted use, distribution, and reproduction in any medium, provided the original author and source are credited.

\section{ABSTRACT}

We investigated the variation of CALR-mutant burden during follow-up in $\mathbf{1 0 5}$ CALR-mutant MPN and compared it to the variation of JAK2-mutant burden in 226 JAK2-mutant MPN.

The median allele burden at last evaluation was significantly higher than at first evaluation in essential thrombocythemia (ET) $(49.5 \%$ vs $45 \%, P<.001)$ but not in primary myelofibrosis (PMF) (52\% vs $51 \%, P$ 0.398). Median values of slope were positive both in ET (0.071) and in PMF (0.032). In CALR-mutant ET there was a difference between natural and therapy-related slope $(P$ 0.006).

In the JAK2-mutated cohort, the median allele burden at last evaluation was not different respect to that at first evaluation, neither in ET $(22.9 \%$ vs $23.2 \%, P=0.216)$

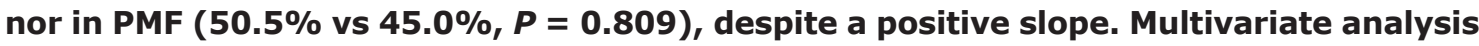
to evaluate the effect of mutation (CALR vs JAK2) on the slope of mutant burden in not treated pts with a positive slope adjusting for diagnosis (ET vs PMF) showed a trend toward a higher increase of mutant burden in CALR vs JAK2 ( $\beta=0.19, P=0.061)$ with no difference between diagnosis $(P=0.419)$. The findings of this study suggest that clonal expansion in CALR-mutant MPN is faster than that observed in JAK2-mutant MPN.

\section{INTRODUCTION}

The three classic Philadelphia-negative myeloproliferative neoplasms (MPN), polycythemia vera $(\mathrm{PV})$, essential thrombocythemia (ET) and primary myelofibrosis (PMF), are clonal hematopoietic disorders that cause expansion of one or more myeloid lineages [1].

Most patients with ET or PMF not associated with $J A K 2$ or $M P L$ alterations carry somatic mutations of $C A L R$ $[2,3]$. The clinical course of $C A L R$-mutated patients is more indolent than that of $J A K 2$-mutated patients, having a lower risk of thrombosis in ET and a better survival in PMF [4-7]. Previous studies have reported that most CALR mutations are heterozygous $[2,3]$.

Data regarding sequential evaluations of CALRmutant burden during follow-up are still lacking. In this study we aimed to investigate the variation of CALRmutant burden during follow-up in CALR-mutant MPN and to compare it to the variation of $J A K 2 \mathrm{~V} 617 \mathrm{~F}$-mutant burden in JAK2-mutant MPN matched for diagnosis.

\section{RESULTS AND DISCUSSION}

All 105 CALR-mutated patients remained positive at second evaluation: among the five secondary acute myeloid leukemia (AML) evolved from CALR-mutant MPN no cases of $C A L R$ negative AML was observed. This is in contrast with the observation that JAK2-mutant MPN frequently yields $J A K 2$ negative AML $[8,9]$.

In $C A L R$-mutant ET the median allele burden at last evaluation was higher than at first evaluation $(49.5 \%$, range $9-94 \%$ vs $45 \%$, range $3 \%-95 \%, P<.001)$. In $C A L R$ - 
mutant PMF the median allele burden at last evaluation (52\%, range $20-75 \%$ ) did not significantly differ from the median allele burden at first evaluation $(51 \%$, range $7-60 \%, P$ 0.398). CALR allele burden at first and last evaluation in ET and PMF are reported in Figure 1.

Median values of slope were positive both in ET (0.071 increase of CALR mutant alleles/month, range $-1.114 ; 2.789)$ and in PMF $(0.032$ increase of CALR mutant alleles/month, range $-5.526 ; 1.229)$, without a significant difference between the two diagnosis $(P 0.319)$. Taken together these data suggest a progressive increase of the clone during time.

Type 1-like and type 2-like CALR mutations did not differ in terms of baseline values and evolutionary pattern. The baseline values did not differ both considering only the subgroup of patients assessed at clinical diagnosis (35\% in type 1 -like vs $38.5 \%$ in type 2 -like, $P 0.863$ ) and considering all patients (48.5\% in type 1 -like vs $46.5 \%$ in type 2-like, $P$ 0.463). Also the slope was not different (0.060 in type 1-like, 0.059 in type 2 -like, $P 0.340$ ).

The delta CALR (difference between last and first $C A L R$ allele burden evaluation) according to time interval between first and last evaluation in ET and PMF is reported in Figure 2.

To investigate whether cytoreductive treatment might influence the mutant allele burden we divided patients in "Treated" (72 patients) if they had received at least one cytoreductive treatment before or between the two sequential evaluations and "Untreated" (33 patients) if they did not receive any treatment. Mann Whitney $U$ test showed a difference between natural $(0.178)$ and



Figure 1: $\boldsymbol{C A L R}$ allele burden. $C A L R$ allele burden (\%) at first and last evaluation in patients with essential thrombocythemia (ET) and primary myelofibrosis (PMF). therapy-related slope $(0.015)(P$ 0.005) in CALR-mutated patients. When repeating the same analysis in the two disorders separately, we confirmed a difference between natural $(0.207$, range $-0.595 ; 2.789)$ and therapy-related not in PMF $(0.033$, range $-0.138 ; 1.229$ vs 0.012 , range $-5.526 ; 1.22, P 0.451)$. The slope of CALR allele burden in treated and untreated ET and PMF is reported in Figure 3. To evaluate whether the difference between natural and therapy-related slope observed in ET might be due to the effect of interferon on the allele burden we compared the slope of ET patients treated with interferon $(N=7)$ with the slope of ET patients treated with other cytoreductive drugs $(N=45)$ but we did not find a statistically significant difference $(P=.0883)$. Two CALR-mutated patients underwent allogeneic bone marrow transplantation (BMT) and obtained complete molecular remission at the first evaluation performed 100 days after BMT.

Then we evaluated whether the increase of the allele burden in ET patients might depend upon disease evolution. We compared the slope of ET patients with disease evolution, including 6 patients who developed post-ET myelofibrosis and 3 patients who progressed to acute myeloid leukemia, with the slope of ET patients without disease evolution. We did not observe a difference between ET patients who developed and those who did not develop disease progression, both in patients with positive slope $(P$ 0.329) and in patients with negative slope $(P$ 0.280). The expansion of the CALR-mutated clone during time seems to occur independently from disease progression.

PMF slope $(0.015$, range $-1.114 ; 2.352)$ in ET $(P 0.006)$ but 
It has been previously shown that patients with high CALR-mutant burden had acquired copy neutral loss of heterozygosity of chromosome $19 \mathrm{p}$, involving transition from heterozygosity to homozygosity for the CALR mutation [2]. At any rate $19 \mathrm{pLOH}$ appears to be relatively uncommon event [4], in contrast with $9 \mathrm{pLOH}$ in JAK2-mutant MPN and $1 \mathrm{pLOH}$ in $M P L$-mutant MPN $[10,11]$. Accordingly, in our study cohort, only five CALRmutated patients had a mutant allele burden equal or greater than $75 \%$. In addition, the genotyping of the rs1049481 SNP in these cases was not informative in four patients, because of homozygosity both in granulocytes and in T lymphocytes. The single informative patient (heterozygous in T lymphocytes) showed homozygous granulocytes, thus confirming $19 \mathrm{pLOH}$, likely as consequence of uniparental disomy, as previously shown [2].

Then we repeated the same analysis in a cohort of 226 $J A K 2$-mutated patients with similar diagnosis. The median allele burden at last evaluation was not different respect to that at first evaluation, neither in ET (22.9\%, range $1 \%-99.9 \%$ vs $23.2 \%$, range $1.7-97.6 \%, P=0.216)$ nor in PMF (50.5\%, range $1 \%-99.1 \%$ vs $45 \%$, range $1.5-98.6 \%$, $P=0.926$ ), although both $J A K 2$-mutant ET and $J A K 2-$ mutant PMF showed a positive slope $(0.007$ and 0.012 respectively). This might suggest that the clonal expansion in CALR-mutant MPN is faster than that observed in JAK2mutant MPN. To test this hypothesis and to evaluate the effect of the type of mutation (CALR vs $J A K 2$ ) on the slope of mutant burden adjusting for the effect of diagnosis (ET vs PMF), we performed a multivariate linear regression in all untreated patients (to avoid the potential influence of cytoreduction). Among patients with a positive slope, we observed a trend toward a higher increase of mutant burden in $C A L R$ vs $J A K 2(\beta=0.19, P=0.061)$ with no difference between diagnosis $(P=0.419)$, as reported in Figure 4 .

Taken together, these results suggest that the CALRmutant stem cell would achieve clonal dominance much faster than the $J A K 2$-mutant one and confirm the model initially proposed [12] in which the clonal evolution of $C A L R$-mutant MPN appears to be mainly associated with a progressive expansion of a mutant heterozygous clone that eventually becomes fully dominant in the bone marrow. Anyway, the sequential evaluation of CALR-mutant burden is not recommended in the clinical practice as it lacks clinical utility.

\section{MATERIALS AND METHODS}

Inclusion in the current study required a diagnosis of ET or PMF, availability of clinical data at diagnosis and during follow-up and at least two DNA samples to assess variations of mutant allele burden.

We identified in our database a total of 105 consecutive patients with CALR-mutant ET and PMF (76 patients affected with ET, 29 patients affected with PMF). This cohort was compared with a cohort of $226 J A K 2$ V617F-mutated consecutive MPN patients with similar diagnosis (183 patients affected with ET, 43 patients affected with PMF). The two cohorts were diagnosed and followed at the Department of Hematology

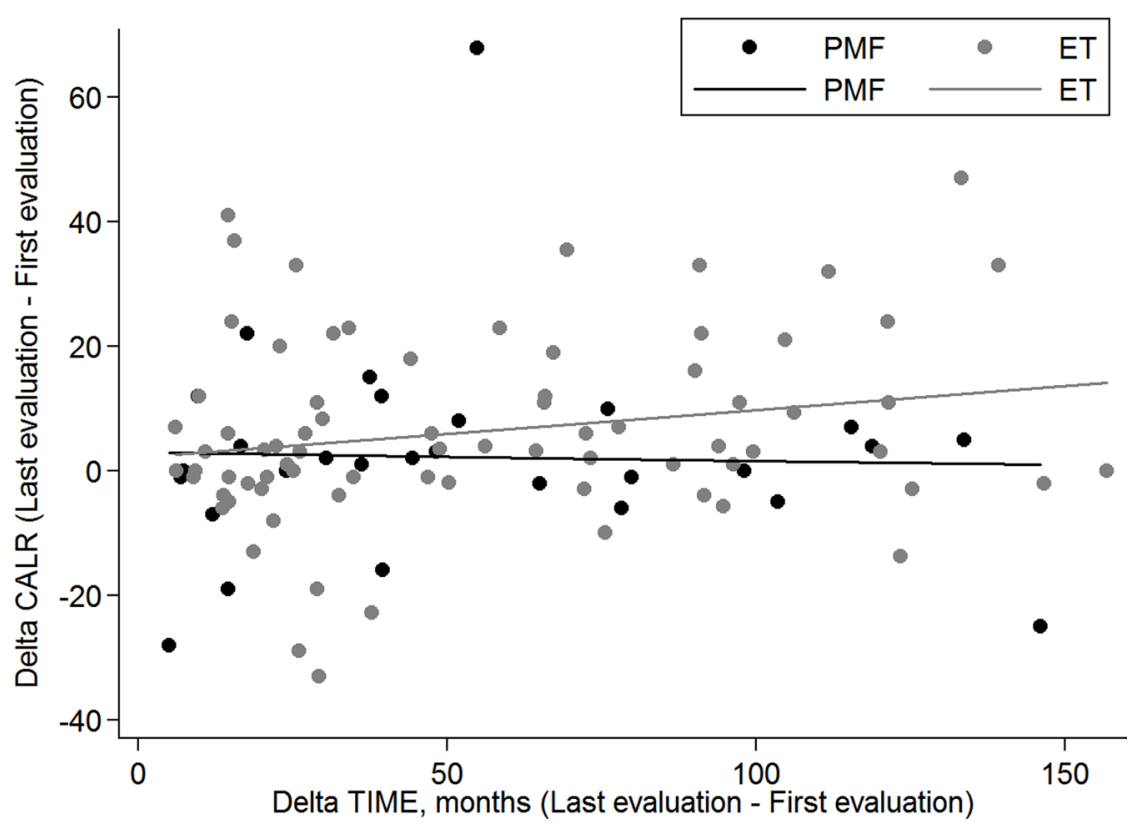

Figure 2: Scatter plot of Delta $\boldsymbol{C A L R}$ according to Delta time. The Delta $C A L R$ (difference between last and first $C A L R$ allele burden evaluation) according to Delta time (time between last and first evaluation) in ET (grey dots) and PMF (black dots). In PMF patients the Delta CALR does not significantly change with the increasing of interval time between first and last evaluation (beta $=-0.01 P=.866$ ); in ET patients the Delta CALR increases (with borderline significance) with the increasing of interval time between first and last evaluation (beta $=0.08 P=.074)$. 
Table 1: Clinical characteristics of the $105 C A L R$-mutated patients and $226 J A K 2$-mutated patients

\begin{tabular}{lcccc}
\hline & \multicolumn{2}{c}{ CALR-mutated } & \multicolumn{2}{c}{ JAK2-mutated } \\
& ET $(\boldsymbol{n}=\mathbf{7 6})$ & $\mathbf{P M F}(\boldsymbol{n}=\mathbf{2 9})$ & ET $(\boldsymbol{n}=\mathbf{1 8 3})$ & PMF $(\boldsymbol{n}=\mathbf{4 3})$ \\
\hline $\begin{array}{l}\text { Median age at first evaluation, } \\
\text { years (range) }\end{array}$ & 45 & 46 & 46 & 58 \\
Gender, M/F & $(15-77)$ & $(18-75)$ & $(15-83)$ & $(27-82)$ \\
$(\%)$ & $(55 \% / 45 \%)$ & $(41 \% / 59 \%)$ & $(38 \% / 62 \%)$ & $(65 \% / 35 \%)$ \\
$\begin{array}{l}\text { Median follow-up, years } \\
\text { (range) }\end{array}$ & 8.73 & 8.16 & 9.33 & 6.69 \\
$\begin{array}{l}\text { Median interval between } \\
\text { first-last sample, } \\
\text { (interquartile range) }\end{array}$ & $(1.07-25.76)$ & $(0.71-16.67)$ & $(1.03-28.97)$ & $(0.84-18.43)$ \\
$\begin{array}{l}\text { Median number of blood } \\
\text { samples per patient (range) }\end{array}$ & $(21.4-91)$ & & & \\
\hline
\end{tabular}

Oncology, Fondazione IRCCS Policlinico San Matteo Pavia from 1985 to 2014. Their clinical characteristics are reported in Table 1. This study was approved by the Ethics Committee of Fondazione IRCCS Policlinico San Matteo, Pavia, Italy. The procedures followed were in accordance with the Helsinki Declaration, and samples were obtained with patients' written informed consent. Diagnosis of
MPN was made in accordance with the criteria in use at the time of the first observation.

Granulocytes' DNA from peripheral blood was used for molecular analysis. CALR exon 9 mutations were assessed with Sanger sequencing, as previously reported [4]. The evaluation of $C A L R$-mutant burden was done comparing the height of the mutated and wild type

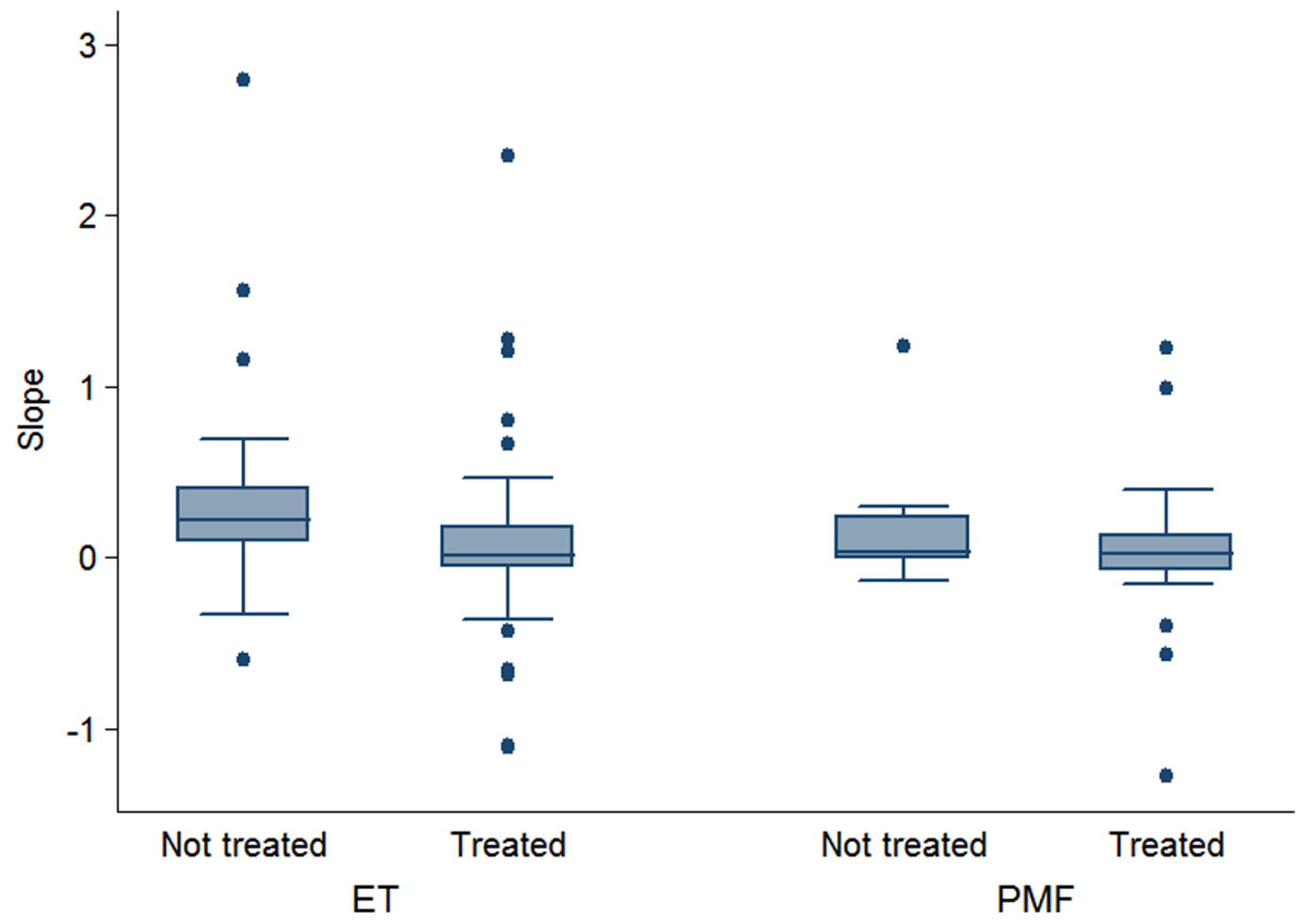

Figure 3: Slope of the $\boldsymbol{C A L R}$ allele burden. Slope of $C A L R$ mutant alleles between sequential evaluations in patients with essential thrombocythemia (ET) and primary myelofibrosis (PMF). The left box-plot shows the natural slope obtained in patients who did not receive cytoreduction; the right box-plot shows the therapy-related slope obtained in patients who receive a myelosuppression. Mann Whitney $U$ test showed a difference between natural and therapy-related slope in ET $(P 0.006)$ but not in PMF $(P 0.451)$ One treated PMF outlier patient $(-5.526)$ was not represented for graphic reasons. 
peaks. To assess the sensitivity of our technique, genomic DNAs from patients with $50 \%$ CALR mutant allelic burden (assessed by fragment length analysis [2]) were diluted into DNA from a healthy control. With the use of $40 \mathrm{ng}$ of matrix DNA, dilutions up to 1:8 for type 1 deletion and 1:16 for type 2 insertion still produced detectable and valuable mutant traces, indicating a sensitivity of the method of approximately $6 \%$ and $3 \%$, respectively. Sanger data were validated in an independent cohort of $98 C A L R$-mutated patients previously evaluated using fragment analysis (median CALR-mutant burden: 47\%; range: 10-100) [2]. This cohort included 34 samples of the study cohort. No false-negative samples were found using Sanger analysis and the comparison of CALR-mutant burden obtained with the two methods showed a very high Pearson's correlation ( $\rho$ 0.94, $P<0.001$ ). Sanger sequencing allowed also the genotyping of the rs1049481 [13].

A quantitative real-time polymerase chain reaction (qRT-PCR)-based allelic discrimination assay was employed for the quantification of the $J A K 2 \mathrm{~V} 617 \mathrm{~F}$ allele $[14,15]$. The different types of CALR mutations were classified as type 1-like and type 2-like according to our previous study [16].

The median interval between first and last sample collection was 45.5 months in ET (interquartile range
21.4-91) and 39.6 months in PMF (interquartile range 17.7-78.2).

Quantitative variables have been summarized in terms of median and range or interquartile range, while categorical variables were described by counts and percentage. The Wilcoxon signed-rank test for paired data was adopted to compare the median value of allele burden at last evaluation respect to the median value at first evaluation. As several patients had more than two sequential evaluations, the variation of allele burden during follow-up was evaluated by the slope of a regression model with allele burden analyzed as outcome and months of follow-up analyzed as independent variable: a slope value higher than 0 refers to an increase of allele burden during time while a negative slope value indicates a decrease of allele burden. The comparison of median slope between two groups was tested by the Mann-Whitney $U$ test for unpaired data. The linear regression model was adopted to evaluate the effect of type of mutation and diagnosis on the slope. $P$-values less than $5 \%$ were considered significant. Statistical analyses were performed using Stata 12.1 (StataCorp. 2011. Stata Statistical Software: Release 12. College Station, TX: StataCorp LP) software.

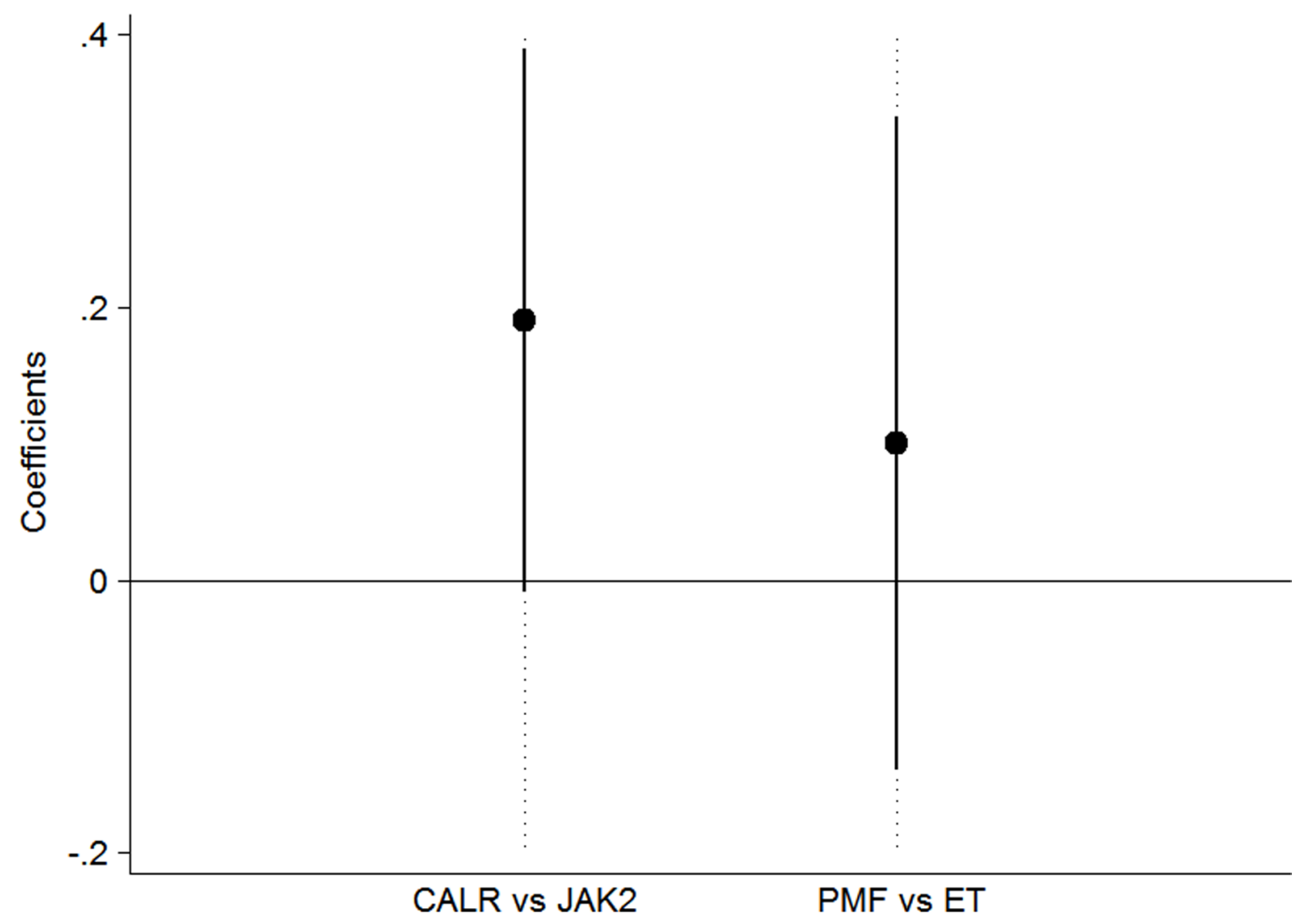

Figure 4: Effect of the mutational status on the slope of mutant burden. Multivariate regression with slope as outcome and mutation and diagnosis as covariates. The analysis was carried on the subgroup of patients with essential thrombocythemia (ET) and primary myelofibrosis (PMF) who were untreated to avoid the potential influence of cytoreduction. There was a trend toward a higher increase of mutant burden in CALR vs JAK2 $(\beta=0.19, P=0.061)$ with no difference between diagnosis $(P=0.419)$. 


\section{Authors' contributions}

$\mathrm{ERu}$ and $\mathrm{CC}$ designed research and wrote the paper; VVF performed statistical analysis; DP performed molecular investigations; ERu, CC, ERo, MB, MC, ICC, $\mathrm{BL}, \mathrm{EF}, \mathrm{DT}, \mathrm{CA}$ collected clinical data; ERu and MC finalized the manuscript.

\section{CONFLICTS OF INTEREST}

The authors declare no competing financial interest.

\section{FUNDING}

This work was supported by a grant from Associazione Italiana per la Ricerca sul Cancro (AIRC; Milan, Italy), Special Program Molecular Clinical Oncology 5x1000 to AIRC-Gruppo Italiano Malattie Mieloproliferative (AGIMM) project $\# 1005$, and by a grant from AIRC (my first AIRC grant MFAG-201415672) to ERu.

\section{REFERENCES}

1. Arber DA, Orazi A, Hasserjian R, Thiele J, Borowitz MJ, Le Beau MM, Bloomfield CD, Cazzola M, Vardiman JW. The 2016 revision to the World Health Organization classification of myeloid neoplasms and acute leukemia. Blood. 2016; 127:2391-2405.

2. Klampfl T, Gisslinger H, Harutyunyan AS, Nivarthi H, Rumi E, Milosevic JD, Them NCC, Berg T, Gisslinger B, Pietra D, Chen D, Vladimer GI, Bagienski K, et al. Somatic Mutations of Calreticulin in Myeloproliferative Neoplasms. New England Journal of Medicine. 2013; 369:2379-2390.

3. Nangalia J, Massie CE, Baxter EJ, Nice FL, Gundem G, Wedge DC, Avezov E, Li J, Kollmann K, Kent DG, Aziz A, Godfrey AL, Hinton J, et al. Somatic CALR mutations in myeloproliferative neoplasms with nonmutated JAK2. N Engl J Med. 2013; 369: 2391-2405.

4. Rumi E, Pietra D, Ferretti V, Klampfl T, Harutyunyan AS, Milosevic JD, Them NCC, Berg T, Elena C, Casetti IC, Milanesi C, Sant'Antonio E, Bellini M, et al. JAK2 or CALR mutation status defines subtypes of essential thrombocythemia with substantially different clinical course and outcomes. Blood. 2014; 123:1544-1551.

5. Rotunno G, Mannarelli C, Guglielmelli P, Pacilli A, Pancrazzi A, Pieri L, Fanelli T, Bosi A, Vannucchi AM. Impact of calreticulin mutations on clinical and hematological phenotype and outcome in essential thrombocythemia. Blood. 2014; 123:1552-1555.

6. Rumi E, Pietra D, Pascutto C, Guglielmelli P, MartinezTrillos A, Casetti I, Colomer D, Pieri L, Pratcorona M, Rotunno G, Sant'Antonio E, Bellini M, Cavalloni C, et al. Clinical effect of driver mutations of JAK2, CALR, or MPL in primary myelofibrosis. Blood. 2014; 124:1062-1069.
7. Tefferi A, Lasho TL, Finke CM, Knudson RA, Ketterling R, Hanson CH, Maffioli M, Caramazza D, Passamonti F, Pardanani A. CALR vs JAK2 vs MPL-mutated or triplenegative myelofibrosis: clinical, cytogenetic and molecular comparisons. Leukemia. 2014; 28:1472-1477.

8. Campbell PJ, Baxter EJ, Beer PA, Scott LM, Bench AJ, Huntly BJ, Erber WN, Kusec R, Larsen TS, Giraudier S, Le Bousse-Kerdilès MC, Griesshammer M, Reilly JT, et al. Mutation of JAK2 in the myeloproliferative disorders: timing, clonality studies, cytogenetic associations, and role in leukemic transformation. Blood. 2006; 108:3548-3555.

9. Theocharides A, Boissinot M, Girodon F, Garand R, Teo SS, Lippert E, Talmant P, Tichelli A, Hermouet S, Skoda RC. Leukemic blasts in transformed JAK2-V617F-positive myeloproliferative disorders are frequently negative for the JAK2-V617F mutation. Blood. 2007; 110:375-379.

10. Kralovics R, Passamonti F, Buser AS, Teo SS, Tiedt R, Passweg JR, Tichelli A, Cazzola M, Skoda RC. A gain-offunction mutation of JAK2 in myeloproliferative disorders. N Engl J Med. 2005; 352:1779-1790.

11. Rumi E, Pietra D, Guglielmelli P, Bordoni R, Casetti I, Milanesi C, Sant'Antonio E, Ferretti V, Pancrazzi A, Rotunno G, Severgnini M, Pietrelli A, Astori C, et al. Acquired copy-neutral loss of heterozygosity of chromosome $1 \mathrm{p}$ as a molecular event associated with marrow fibrosis in MPL-mutated myeloproliferative neoplasms. Blood. 2013; 121:4388-4395.

12. Cazzola M, Kralovics R. From Janus kinase 2 to calreticulin: the clinically relevant genomic landscape of myeloproliferative neoplasms. Blood. 2014; 123:3714-3719.

13. Harutyunyan AS, Jaeger R, Chen D, Berg T, Rumi E, Gisslinger B, Pietra D, Gisslinger H, Cazzola M, Kralovics R. Allelic imbalance in CALR somatic mutagenesis. Leukemia. 2015; 29:1431-1435.

14. Malcovati L, Della Porta MG, Pietra D, Boveri E, Pellagatti A, Galli A, Travaglino E, Brisci A, Rumi E, Passamonti F, Invernizzi R, Cremonesi L, Boultwood J, et al. Molecular and clinical features of refractory anemia with ringed sideroblasts associated with marked thrombocytosis. Blood. 2009; 114: 3538-3545.

15. Passamonti F, Rumi E, Pietra D, Della Porta M, Boveri E, Pascutto C, Vanelli L, Arcaini L, Burcheri S, Malcovati L, Lazzarino M, Cazzola M. Relation between JAK2 (V617F) mutation status, granulocyte activation, and constitutive mobilization of CD34(+) cells into peripheral blood in myeloproliferative disorders. Blood. 2006; 107:3676-3682.

16. Pietra D, Rumi E, Ferretti VV, Di Buduo CA, Milanesi C, Cavalloni C, Sant'Antonio E, Abbonante V, Moccia F, Casetti IC, Bellini M, Renna MC, Roncoroni E, et al. Differential clinical effects of different mutation subtypes in CALR-mutant myeloproliferative neoplasms. Leukemia. 2016; 30:431-8. 